\title{
La Ganadería Pre Hispánica
}

\section{Pre-Hispanic Livestock}

Alvaro Hernán Castañeda Mesía ${ }^{1}$

\section{RESUMEN}

La ganadería tiene como antecedente la domesticación de animales que es el fenómeno fundamental para que se implemente la ganadería como actividad que cría y cuida el ganado. Pero este fenómeno no se hubiera dado si es que el hombre prehispánico no hubiera compartido un mismo espacio de vida con los camélidos la ganadería no se hubiera generado. La ganadería es una actividad que con el devenir histórico que va desde finales del arcaico y el intermedio tardío que se donde se consolida como una actividad que se "define como el arte de criar ganado procurando obtener de él, los mejores resultados prácticos"; esta definición explica la evolución y especialización que tuvo esta actividad. La ganadería como actividad es cíclica y comienza con la trashumancia estacional y culmina con la ganadería comercial que es como la conocemos hoy en día. Esta actividad tiene como característica el pastoreo que se entiende como llevar los ganados al campo en busca de pastos y cuidar de ellos mientras los comen.

Palabras clave: La domesticación, la ganadería y el pastoreo.

\begin{abstract}
The livestock has as antecedent the domestication of animals that is the fundamental phenomenon for livestock to be implemented as an activity that breeds and cares for livestock. But this phenomenon would not have occurred if the pre-Hispanic man had not shared the same space of life with the camelids. The livestock would not have been generated. Livestock is an activity that with the historical evolution that goes from the end of the archaic and the late intermediate that is where it is consolidated as an activity that is "defined as the art of raising cattle trying to obtain from it, the best practical results"; this definition explains the evolution and specialization that this activity had. Livestock as an activity is cyclical and begins with seasonal transhumance and culminates with commercial livestock that is how we know it today. This activity has as a characteristic the grazing that is understood as taking the cattle to the field in search of pastures and taking care of them while they eat them.
\end{abstract}

Keywords: domestication, livestock and grazing.

'Mg. en Ciencias con mención en Gestión Ambiental.Correo electrónico: Alvaro.castaneda@untrm.edu.pe 


\section{INTRODUCCIÓN}

La ganadería prehispánica tiene como antecedente a la domesticación y para ello explicare cuales son los factores que permiten que la domesticación se manifieste; según Valadez, 1987 "el primer factor es el Fáunico no todas las especies soportan los cambios de la domesticación, el segundo factor es el Humano se da cuando el hombre ha evolucionado o sea observa razona y aprende de los animales que le rodean, el tercer y último es el Interés es la razón del por que se llega a domesticar una especie (camélidos)." Cuando estos tres factores se conjugan se da la domesticación y por ende la ganadería. Otro autor que reafirma el criterio del humano y su interés sobre el uso de animales para domesticarlos en lo que concierne a sus hábitos reproductivos es Renfrew/Van, 1991 "que dice que un criterio sobre la domesticación animal concierne a la interferencia humana con los hábitos de reproducción natural de ciertas especies, lo cual ha conducido a cambios en las características fisicas de aquellas especies en su estado silvestre." Por eso no todas las especies soportan el proceso de domesticación por los cambios en sus características físicas y de reproducción. Se suma a lo dicho el haber compartido habitads con los camélidos y por ello el conocimiento de los hábitos para poderlo domesticar, y con el interés que va entorno a obtener sus alimentos de forma más sencilla ya que cría al animal. (Ver foto 01)

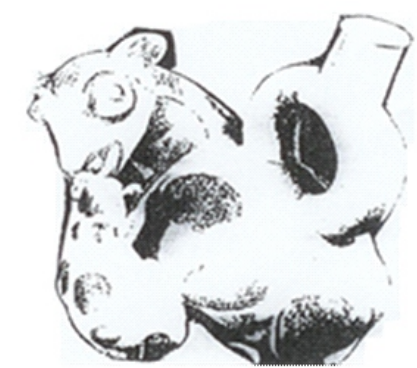

Foto 01 Actividades de los camélidos

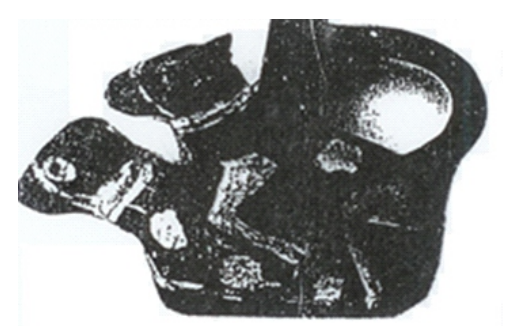

Foto 02 Actividades de los camélidos

(Shimada. A y Melody, 1981)

\section{MATERIAL Y MÉTODO}

En la introducción mencionamos a la domesticación como elemento generador de la ganadería y por ende tenemos que tomar la ganadería como un proceso cultural y para ello tomaremos como cronología el espacio comprendido desde el arcaico hasta el intermedio tardío. En donde el primer objetivo es probar que los camélidos fueron domesticados en diferentes pisos altitudinales y regiones que van desde la costa hasta la amazonia. Y el segundo objetivo es explicar arqueológicamente la evolución de la ganadería tomando algunos ejemplos y evidencias encontradas. En sitios aún más tempranos y así poder explicar este proceso cultural.

\section{RESULTADOS}

Los resultados de esta investigación que tienen continum cultural y por ello analizaremos a dos pueblos de cazadores; uno es Lauricocha que según Cardich 1958 "La economía que indican los restos de los tres primeros horizontes (Lauricocha I, Lauricocha II y Lauricocha III) es la que corresponde a cazadores superiores. Los abundantes fragmentos de huesos, algunos trabajados, pero en gran parte solamente residuos de alimentación, pertenecen a llamas (Lama glama), guanaco (Lama guanicoe), vicuñas (Vicugna vicugna), tarucas o tarugos (Hippocamelus antisensis) y algunos cérvidos; muchos fragmentos, que corresponderían a animales de tamaños similares a los nombrados, no han podido ser clasificados aún. La escasez de huesos de aves y roedores, como residuos de alimentación, nos indican que aquellos cazadores no tuvieron dificultades en procurarse alimentos." $Y$ el otro es Telarmachay que de acuerdo con Lavallee, 1995 "Entre 9000 y 7200 $\mathrm{BP}$, aproximadamente, se practica la caza generalizada de todos los ungulados de la puna, que evoluciona, entre 7200 y 6000 BP, hacia una caza especializada de guanacos y vicuñas, hasta la aparición entre 6000 y 55000 BP aproximadamente, de los primeros animales domésticos y, por último, la crianza de animales plenamente domésticos después de 5500 BP. Entre la ocupación más antigua del sitio, correspondiente a la fase VII, y que se inicia hacia 9 $000 \mathrm{BP}$, y la última ocupación pre-cerámica de la fase IV que se termina hacia $3800 \mathrm{BP}$, se observa una diferencia acumulativa de $-24,27 \%$ de cérvidos frente a $+23,91 \%$ de camélidos." Como se ve en las citas de Cardich y Lavallee estos pueblos fueron cazadores de camélidos y de otros animales como los cérvidos, siguiendo con esta secuencia hablaremos de un sitio del Arcaico Superior con Influencia en el formativo como lo afirma Morales Chocano, 1974 "San Blas, es uno de estos focos pero no de Chavín 
sino más bien de Kotosh, la puna brinda a estas sociedades las condiciones necesarias para el desarrollo de la crianza y pastoreo de rebaños de auquénidos evidenciados en Huarmipuquio, Huaychascoto, Ondores, Parí y el mismo San Blas, donde a su vez existe el elemento básico que es la Sal. Hemos encontrado testimonios de origen selvático, de alfarería del Valle de Higueras que migran hacia las punas." El siguiente sitio formativo combina la ganadería y la agricultura como lo establece Sachun, 1986 "el Periodo Layzon: La presencia de camélidos, el cultivo permanente de tres vegetales; maíz, frejol y una especie de leguminosa tipo arveja y el consiguiente desplazamiento de los servidos, permitiendo a la vez el medio de transporte a base de las llamas, ello nos permite avizorar el eficiente control y manejo de los diferentes nichos ecológicos, tanto del valle de Cajamarca así como la relación recíproca con la zona chala y yunga." Otro sitio que prueba la aclimatación de los camélidos a la costa como lo define Altamirano, 1987 "La llama costeña de Bayovar ya estaba aclimatada al habitad desértico debido a la necesidad de transporte, consumo y a la prolongada adaptabilidad que data desde el formativo. El camélido costeño estaba muy difundido y era común verse entre los poblados nucleares y lejanos Chimú. Por lo que la llama jugó un papel muy importante en la vida cotidiana de los yungas" (ver foto 03).

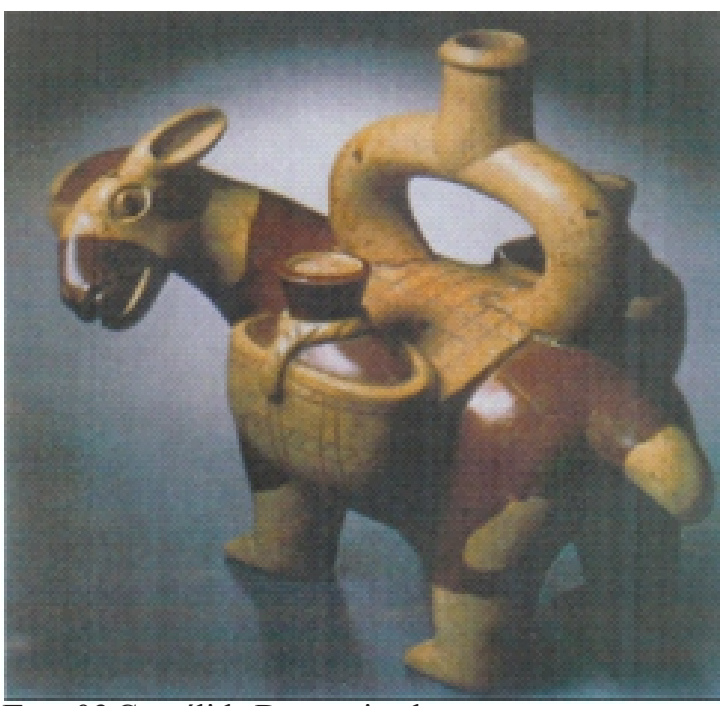

Foto 03 Camélido Domesticado

National Biographic.

En cuanto al intermedio tardío en lo relacionado a lo Inca Valcárcel, 1984:11-19 “afirma lo siguiente; En cuanto al ganado, hízose una división semejante a la tierra, señalándose pastos y animales para el Inca, la iglesia y la comunidad, observándose la misma regla que para los productos. Al ganado del Inca se la llamaba Capac Llama y a los de la comunidad
Guaccha Llama. Púsose mucha diligencia en la conservación y multiplicación del ganado, prohibiéndose la muerte de los animales hembras. Anualmente se hacía la trasquila de la lana de los auquénidos y cada familia recibía la cantidad necesaria para hilar y tejer su ropa, siendo muy vigilada esta labor, castigándose a los negligentes. De la lana del ganado del Inca se tejía para él y su corte, en especial la rica tela llamada Cumbi, la corriente era denominada Abasca. La lana sobrante era almacenada. Todos los depósitos, tanto de comestibles como ropa, fueron hallados por los españoles llenos hasta el tope" (Ver foto 04).

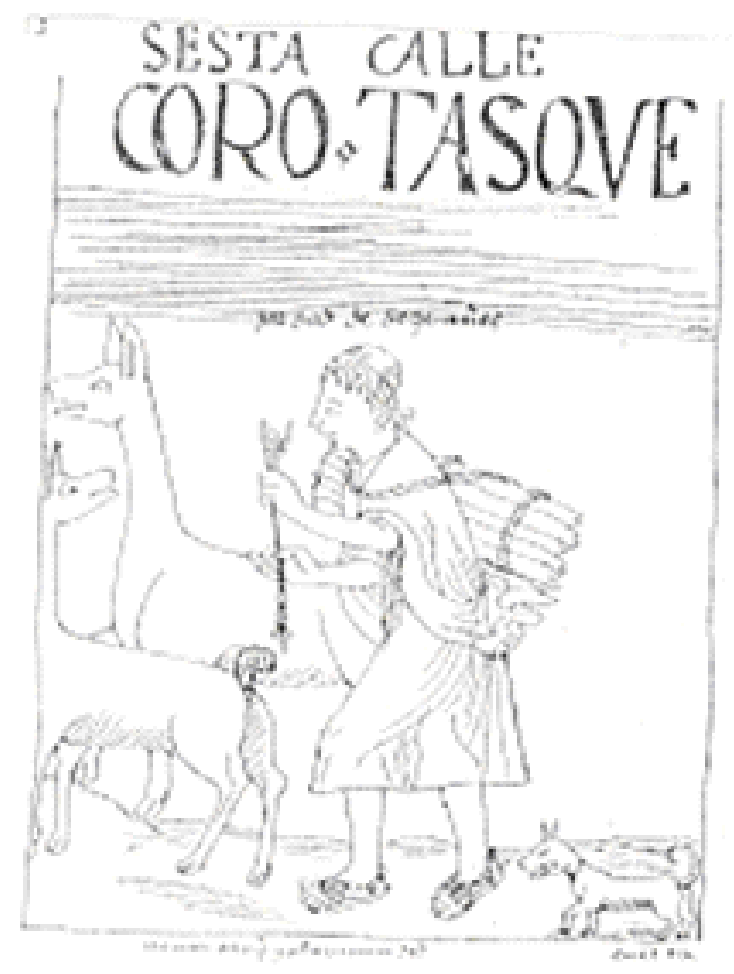

Foto 04 Dibujo de Huamán Poma

\section{DISCUSIÓN}

La ganadería prehispánica es un proceso que define los asentamientos prehispánicos y permite el desarrollo de actividades de intercambio a larga distancia y a la vez permite que el control de los pisos altitudinales sea vertical que va desde las zonas más altas hasta los valles bajos y la costa. Esta actividad fue realizada por un especialista que permitió que esta actividad se desarrolle y nos referimos al pastor que es el que se encarga del cuidado y cría del ganado. Muchos pueblos tuvieron a la ganadería como una actividad que permitió el intercambio con los lugares lejanos y productos de diferente naturaleza desde frutos y cereales hasta textiles y cerámica. Este proceso de intercambio de productos comenzó con 
los grandes centros ceremoniales del formativo y el arcaico que recibían productos desde grandes distancias como la costa y la selva, esto se intensifico durante los desarrollos regionales tardíos hasta lo Wari e Inca.

Debo recalcar que la ganadería fue una actividad que tuvo como origen la caza que permite el conocimiento de la presa y ello nos lleva al conocimiento de los hábitos reproductivos hasta la época de cría, ello se muestra en las fotos 01 y 02 en donde se ve al camélido teniendo relaciones sexuales y cuando ha parido una cría, con el tiempo el camélido se convirtió en una bestia de carga que permitió la conquista de los diferentes pisos altitudinales en forma transversal como lo afirman algunos autores, esto se muestra en la foto 03; si se sigue dentro de la evolución cultural llegaremos a los Incas en donde ya había una tecnología ganadera que permitió que esta actividad se generalice en todo el imperio y se pueda contar con rebaños por millares esto se muestra en la foto 04; si uno junta todas las fotos se puede ver el proceso cultural que va desde el arcaico hasta la época Inca.

\section{CONCLUSIONES}

La primera conclusión nos dice que los camélidos convivieron con los grupos de cazadores recolectores que dieron origen a su domesticación y posteriormente la ganadería como lo ha evidenciado en San Blas y Layzon.

La segunda conclusión es que la ganadería permitió que muchos pueblos tengan un intercambio comercial como base de su economía y no necesitan la agricultura como única forma de subsistencia.

La tercera conclusión es que la ganadería fue muy extendida en los andes en diferentes pueblos y que su punto máximo fue el periodo Inca en donde esta actividad tuvo una gran importancia económica y ritual, ya que este animal se convirtió en una divinidad en los pueblos andinos.

La cuarta conclusión es que después de este pequeño recorrido histórico cultural se evidencia la domesticación del camélido a diferentes climas y geografías.

La quinta conclusión es que dentro de la ganadería existen otras actividades como la elaboración de instrumentos óseos; como agujas, punzones, alisadores y otros instrumentos, otra actividad fue la textileria y se suma a ella la elaboración de sogas con las tripas de los camélidos.

\section{REFERENCIAS BIBLIOGRÁFICAS}

Altamirano Enciso, Alfredo José

1979 “Guía Osteológica de camélidos Sudamericanos" en Serie de Investigaciones $\mathrm{N}^{\mathrm{o}}$ 4, Gabinete de Arqueología Colegio Real U.N.M.S.M. Lima

1983 Historia de los camélido en América pág 2-13 Lima

1983 "Pesca y Utilización de los Camélidos en Manchan" en Boletín de Lima N³0 Año 5 pág. 66

1987 "Restos de Camélidos prehispánicos en la Bahía de Bayovar, Piura". En Boletín de Lima No52-Julio Lima.

1995 Función Ritual de camélidos en la Costa Norte; Ofrendas de Pacatnamú Tesis Doctoral de la P.U.C.P. pág 22-41 Lima.

C. Wheeler, Jane

1984 "La domesticación de la alpaca (Lama pacos L) y la llama (Lama glama L) y el Desarrollo de la Ganadería Autóctona en los Andes Centrales".En el Boletín de Lima No 36 año 6 Noviembre. pág 76 Lima.

1999 "Patrones Prehistóricos de Utilización de los camélidos Sudamericanos".En Boletín de Arqueología del P.U.C.P. Pág 298 Lima.

Custred, Glynn

1974 "Llameros y Comercio Interregionales".En Reciprocidad e Intercambio en los Andes Peruanos. Compilador Giorgio Alberti/ Enrique Mayer I.E.P. Pág 253-261-Lima

Flores Ochoa, Jorge A

198 "Clasificación y Nominación de Camélidos Sudamericanos". En La Tecnología en el Mundo Andino de Heather Lechtman y Ana María Soldi. Pág 195-215.Méjico.

1977 Pastores de Puna Uywamichiq punarunakuna Instituto de Estudios Peruanos. Lima

Huaman Poma de Ayala

1936 Nueva Crónica y Buen Gobierno"(Codex péruvien Ilustré) Institut D'ernología.-Paris

Kent, Johnatan

1982 "The Domestication, and Exploitation of the South American Camelid is Methods of Analysis and their Aplication a Circumlacustrines Archeological sites". tesis Doctoral- Departament of Antropology. W.U. Pág.146. -Washington

Kent D., Johnatan; Vasquez Sánchez, Victor, Rosales Than, Teresa

1998 "Pastoreo y Manejo de Camélidos en la época Lambayeque. Datos Zooarqueológicos" en Revista de la Facultad de Ciencias Sociales 
No5 Pág. 258. 1998 U.N.T.

Mejia Xesspe, Toribio

1998 "Kausay, Alimentación de los Indios". En Tecnología Andina de Rogger Ravines. I.E.P. pág 98 Lima

Morales Chocano, Daniel

1974 "Correlación de las Ocupaciones Formativas entre las Punas de Junín y el Valle de Higueras". En el Segundo Congreso Peruano del Hombre y la Cultura Andina del 27 oct-2 de Nov. Pág 58 Lima.

1977 "Excavaciones en las Salinas de San Blas (Junín)". En el Tercer Congreso Peruano del Hombre y la cultura Andina, Editado por Daniel Morales Chocano. Pág 325-334 Lima

Palacios Ríos, Félix

1985 "Tecnología del Pastoreo". En La Tecnología en el Mundo Andino de Heather Lechtman y Ana Maríe Soldi. U.N.A. Mexico

Rosales Than, Teresa

1998 Evidencias Arqueológicas de Crianza de Camélidos en la Costa Norte del Perú. U.NT. Pág 64 Trujillo

Silver, A

1982 "Determinación de la Edad en los Animales Domésticos". En Ciencia en Arqueología de Don Brotherwe. Pág 289-297 Mexico.

Shimada Melidy y Izumi

1981 "Explotación y Manejo de los Recursos Naturales en Pampa Grande, Sitio Moche V. Significado del Análisis Orgánico" En Revista del Museo Nacional Nacional XLV. pág 52. Lima

Sumar Kalinowski, Julio

1992 "Los camélidos Andinos: Pasado Presente y Futuro" en Alma Mater (2), pág. 67-78. U.N.M.S.M. Lima

Vásquez Sánchez, Víctor F.

1995 Determinación Taxonómica de una Muestra Arqueológica de Camelidae del Sitio Cerro Arena-Valle de Moche. F.B.C Trujillo

Valcárcel, Luis E.

1984 Historia del Perú Antiguo: A Través de la Fuente Escrita Editorial Mejia Vaca, Juan. Pág 11-19.Lima

Wing S. Elizabeth

1977 "Caza y Pastoreo Tradicionales en los Andes Peruanos".En Pastores de Puna de Jorge Flores Ochoa. Pág. 125-128 I.E.P Lima. 